ARTICLE

Received 18 Jun 2014 | Accepted 31 Oct 2014 | Published 8 Dec $2014 \quad$ DOl: 10.1038/ncomms6739

\title{
Pressure-induced chemistry in a nitrogen-hydrogen host-guest structure
}

Dylan K. Spaulding ${ }^{1 \dagger}$, Gunnar Weck${ }^{1}$, Paul Loubeyre ${ }^{1}$, Fréderic Datchi ${ }^{2}$, Paul Dumas ${ }^{3}$ \& Michael Hanfland ${ }^{4}$

New topochemistry in simple molecular systems can be explored at high pressure. Here we examine the binary nitrogen/hydrogen system using Raman spectroscopy, synchrotron X-ray diffraction, synchrotron infrared microspectroscopy and visual observation. We find a eutectic-type binary phase diagram with two stable high-pressure van der Waals compounds, which we identify as $\left(\mathrm{N}_{2}\right)_{6}\left(\mathrm{H}_{2}\right)_{7}$ and $\mathrm{N}_{2}\left(\mathrm{H}_{2}\right)_{2}$. The former represents a new type of van der Waals host-guest compound in which hydrogen molecules are contained within channels in a nitrogen lattice. This compound shows evidence for a gradual, pressure-induced change in bonding from van der Waals to ionic interactions near $50 \mathrm{GPa}$, forming an amorphous dinitrogen network containing ionized ammonia in a room-temperature analogue of the Haber-Bosch process. Hydrazine is recovered on decompression. The nitrogen-hydrogen system demonstrates the potential for new pressure-driven chemistry in high-pressure structures and the promise of tailoring molecular interactions for materials synthesis.

\footnotetext{
${ }^{1}$ CEA, DAM, DIF, F-91297, Arpajon, France. ${ }^{2}$ Institut de Minéralogie, de Physique des Matériaux, et de Cosmochimie (IMPMC), Sorbonne Universités-UPMC Université Paris 6, CNRS UMR 7590, IRD UMR 206, MNHN, 4 place Jussieu, F-75005 Paris, France. ${ }^{3}$ SOLEIL, L'Orme des Merisiers, Saint-Aubin-BP48, Gifsur-Yvette 91192, France. ${ }^{4}$ European Synchrotron Radiation Facility, Grenoble F-38043, France. † Present address: Department of Earth and Planetary Science, Harvard University, 20 Oxford St, Cambridge, Massachusetts 02138, USA. Correspondence and requests for materials should be addressed to D.K.S. (email: dylanspaulding@gmail.com).
} 
T he chemistry and equations of state of simple molecular systems (for example, $\mathrm{N}_{2}, \mathrm{H}_{2}, \mathrm{H}_{2} \mathrm{O}, \mathrm{CO}_{2}, \mathrm{CH}_{4}$ and so on) in the condensed state are of great importance to planetary astrophysics, for the accurate characterization of energetic reaction products and for engineering new molecular compounds by means of pressure-induced chemistry. These are also model systems for understanding chemical bonding, testing fundamental condensed matter theory and probing potential quantum effects at high density.

Extensive studies have shown that pure $\mathrm{N}_{2}$ and pure $\mathrm{H}_{2}$ demonstrate subtle and complex high-pressure behaviour. In solid $\mathrm{N}_{2}$, quadrupole-quadrupole interactions give way to partially and later fully ordered states with increasing pressure, leading to a rich polymorphism at low-to-moderate pressures ${ }^{1}$. In contrast, structural changes in $\mathrm{H}_{2}$ are determined by the interplay between quantum effects and orientational ordering, keeping the molecular centers of mass on an hcp lattice over a very large pressure domain ${ }^{2}$. Recent work on $\mathrm{N}_{2}$ and $\mathrm{H}_{2}$ has focused particular attention on the role of structure in facilitating transitions to nonmolecular and metallic phases at ultra-high pressure. A polymeric form of $\mathrm{N}_{2}$ has been observed above $100 \mathrm{GPa}$ and at high temperature, and may be an ideal highenergy density material if recoverable at ambient conditions ${ }^{3-6}$. In addition, metallic $\mathrm{H}_{2}$ is expected to demonstrate near roomtemperature superconductivity and would represent the ultimate hydride, but has thus far eluded experimental efforts ${ }^{7,8}$.

Despite their fundamental nature and ubiquity, there has yet to be a comprehensive study of the full $\mathrm{N}_{2}-\mathrm{H}_{2}$ binary system, although previous studies have examined limited individual concentrations $s^{9,10}$. Concentration opens a new parameter space (along with pressure and temperature) for exploring new stoichiometric compounds, reactivity in the solid solution and potential paths for the synthesis and stabilization of phases with technological applications. This could lead to new $\mathrm{H}_{2}$-rich compounds for fuel cell technology ${ }^{11}$ or enable $\mathrm{H}_{2}$ metallization by confinement in a host lattice ${ }^{12}$.

The formation of van der Waals compounds containing $\mathrm{N}_{2}$ and $\mathrm{H}_{2}$ demonstrates the potential for new stoichiometries at modest pressures. The first such compounds were produced in combination with rare gases, $\operatorname{Ar}\left(\mathrm{H}_{2}\right)_{2}$ (ref. 13) and $\left(\mathrm{N}_{2}\right)_{11} \mathrm{He}$ (ref. 14). $\mathrm{H}_{2}$ has since been shown to form a family of compounds, including $\left(\mathrm{O}_{2}\right)_{3}\left(\mathrm{H}_{2}\right)_{4}$ (ref. 15), $\mathrm{CH}_{4}\left(\mathrm{H}_{2}\right)_{2}$ (ref. 16), $\mathrm{Xe}\left(\mathrm{H}_{2}\right)_{7}$ (ref. 17) and $\mathrm{SiH}_{4}\left(\mathrm{H}_{2}\right)_{2}$ (ref. 18). There is obvious interest in manipulating pressure-driven chemistry to transform the weak van der Waals interactions into stronger bonds, increasing the likelihood of recovering high-pressure phases at ambient conditions.

Here, we examine the $\mathrm{N}_{2}-\mathrm{H}_{2}$ binary phase diagram at $300 \mathrm{~K}$ to investigate both the chemistry and stability of the high-pressure solid phases. We identify two new van der Waals compounds, $\left(\mathrm{N}_{2}\right)_{6}\left(\mathrm{H}_{2}\right)_{7}$ and $\mathrm{N}_{2}\left(\mathrm{H}_{2}\right)_{2}$. We study their behaviour using a combination of single-crystal X-ray diffraction in the diamond anvil cell (DAC) and Raman and infrared (IR) spectroscopy to characterize the structural, vibrational and chemical properties. $\left(\mathrm{N}_{2}\right)_{6}\left(\mathrm{H}_{2}\right)_{7}$ presents an unexpected host-guest structure, in which an $\mathrm{N}_{2}$ lattice hosts clusters of $\mathrm{H}_{2}$ molecules. This compound shows evidence for a marked change from van der Waals to ionic interactions at $50 \mathrm{GPa}$ and room temperature- a regime in which both end-member molecular species are otherwise quite stable. This unique pressure-induced change in bonding appears to be facilitated by the novel host-guest structure, overcoming the strong $\mathrm{N}_{2}$ triple bond at relatively modest conditions.

\section{Results}

Binary phase diagram. In this work, some 30 samples were prepared at 15 different concentrations to examine the full stoichiometric phase space as a function of pressure and to test thoroughly for reproducibility and chemical equilibrium in several regions of interest. Phase transitions were identified primarily by visual observation based on the difference in refractive index between phases, accompanied by Raman spectroscopy (described further below).

We identify a eutectic-type phase diagram with at least three triple points (near 35, 60 and $80 \mathrm{~mol} \% \mathrm{H}_{2}$ concentration) and two stoichiometric van der Waals compounds at $54 \mathrm{~mol} \%$ and 66 $\mathrm{mol} \% \mathrm{H}_{2}$ concentration, respectively (Fig. 1). The liquidus line

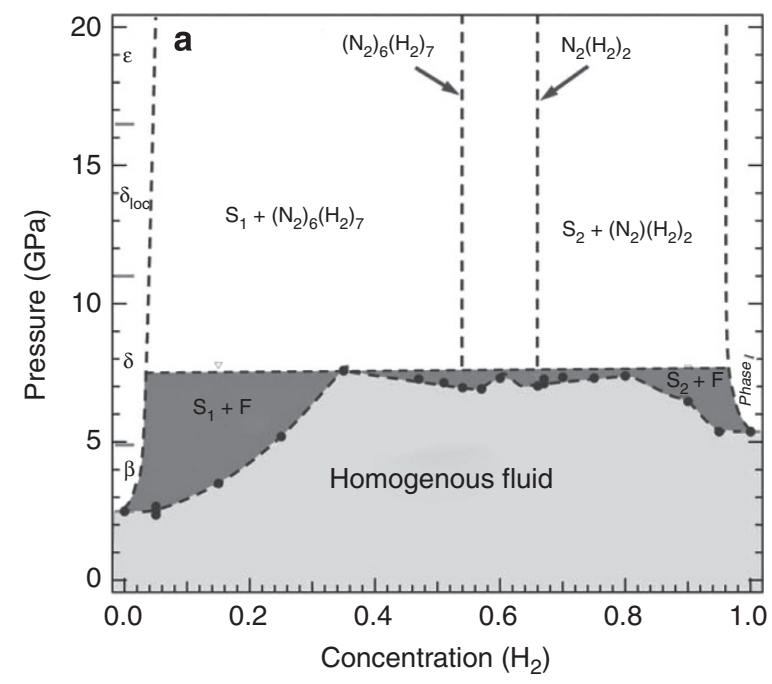

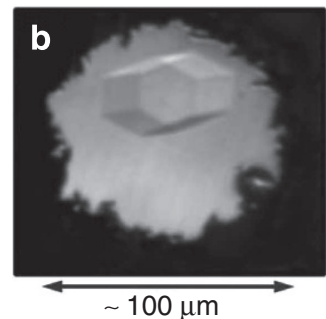
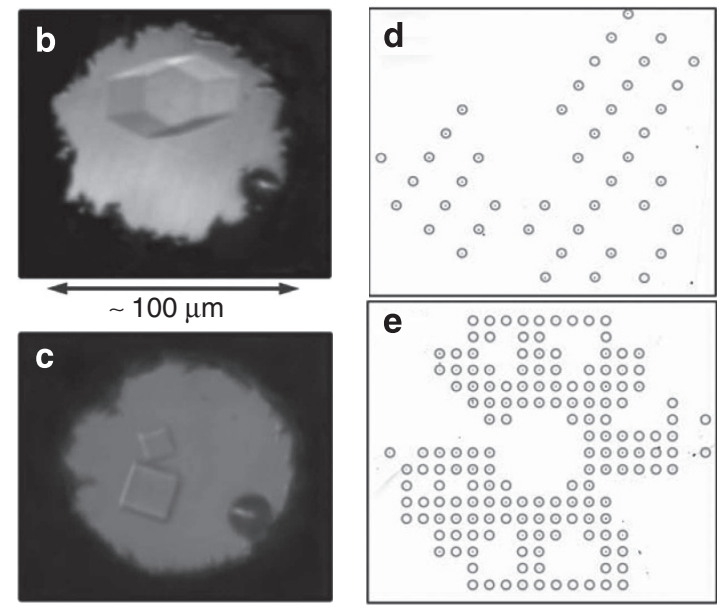

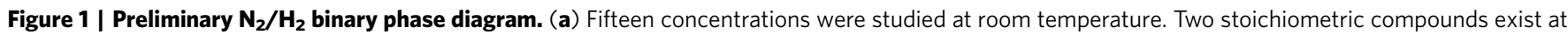
$54 \%$ and 66 mol\% $\mathrm{H}_{2}$ concentration, respectively. Away from these concentrations, phase separation occurs between the van der Waals compounds and either a nitrogen-rich solid (S1) or a hydrogen-rich solid (S2). Data points represent visual observation of complete melting. S $+\mathrm{F}$ indicates solid-fluid

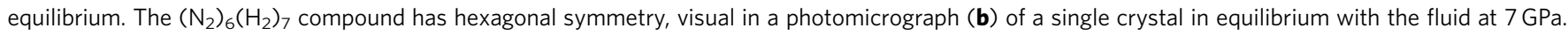
Similarly, single crystals of the $\mathrm{N}_{2}\left(\mathrm{H}_{2}\right)_{2}$ solid are seen to be cubic as they grow in equilibrium with the fluid, (c). Black spheres in the lower right are

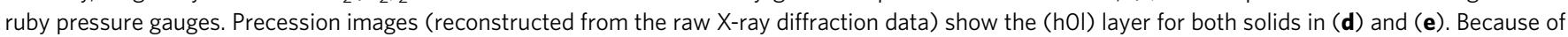
the small mosaicity of the crystals and high detector resolution, the individual points are small and therefore emphasized with circles. 
was determined by visual identification of the disappearance of single crystals and is represented by the locus of data points in Fig. 1a. The two species are completely miscible in the fluid phase, but have a low mutual solubility in the solid phase $-<2$ mol\% $\mathrm{N}_{2}$ in solid $\mathrm{H}_{2}$ and $<5 \mathrm{~mol} \% \mathrm{H}_{2}$ in solid $\mathrm{N}_{2}$. The shape of the liquidus, along with visual observation, confirms the existence of two stoichiometric compounds distinguished by their disparate hexagonal and cubic symmetry (Fig. 1b,c). Continuous pressure measurements during decompression to the fluid state at their precise stoichiometries confirm that they melt congruently.

Structural study. We identify these compounds as $\left(\mathrm{N}_{2}\right)_{6}\left(\mathrm{H}_{2}\right)_{7}$ and $\mathrm{N}_{2}\left(\mathrm{H}_{2}\right)_{2}$, respectively, on the basis of their $\mathrm{H}_{2}$ concentrations and single-crystal X-ray diffraction analyses. Samples were prepared as close to the ideal stoichiometries as possible such that the single-crystals could be grown to fill the sample chamber. In most cases, crystals were allowed to anneal for at least $24 \mathrm{~h}$ at room temperature and 1-2 GPa above the solidus before spectra were recorded. The single crystals are observed to have a small mosaicity in the $\mathrm{X}$-ray data $\left(0.05^{\circ}\right.$ in the detector plane), indicating that they are of high quality.

X-ray diffraction reveals that the $\mathrm{N}_{2}\left(\mathrm{H}_{2}\right)_{2}$ compound (Fig. 1c) crystallizes in a cubic structure with the most probable space groups being $P m-3 m$ and $P m-3 n$. This is reminiscent of the cubic structure of the $\delta$-phase of $\mathrm{N}_{2}(P m-3 n)$ at similar pressures, but with a lattice parameter nearly twice as large $(a=12.349 \AA$ at 9.2 $\mathrm{GPa}$, compared with $6.112 \AA$ for $\delta-\mathrm{N}_{2}$ at slightly lower
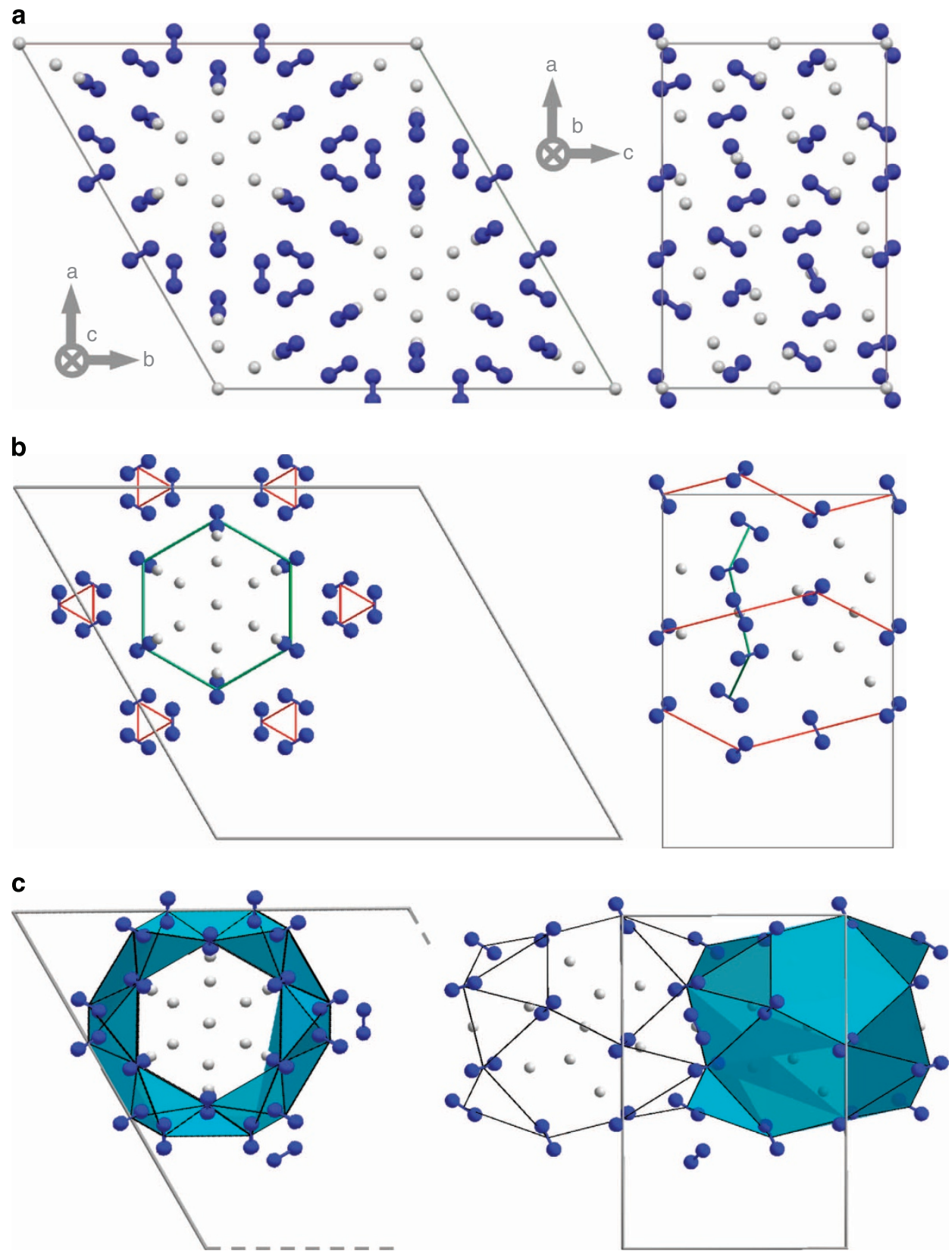

Figure 2 | Structure of the $\left(\mathbf{N}_{\mathbf{2}} \mathbf{~}_{\mathbf{6}}\left(\mathbf{H}_{\mathbf{2}} \mathbf{}_{\mathbf{7}}\right.\right.$ van der Waals compound. (a) Single-crystal X-ray diffraction reveals that clusters of $\mathrm{H}_{2}$ molecules (shown in grey) are confined in channels within an $\mathrm{N}_{2}$ lattice (blue dumbbells). (b) Helical chains of $\mathrm{N}_{2}$ (emphasized in red) orient intermediate $\mathrm{N}_{2}$ molecules in a puckered, hexagonal arrangement (emphasized in green) roughly in the $<001>$ plane. (c) This generates a channel comprised of cage-like volumes along the c-axis, each of which hosts $15 \mathrm{H}_{2}$ molecules. Two of these are shared with the neighbouring volumes. Complete crystallographic data are included in Supplementary Table 1. 
pressure $)^{19,20}$. A complete structural determination could not be achieved on the basis of the present data because of the rapid decrease of the reflection intensities with the diffraction angle, which we believe is due to orientational disorder of the molecules, as in $\delta-\mathrm{N}_{2}$. Despite potential similarities with the case of $\operatorname{Ar}\left(\mathrm{H}_{2}\right)_{2}$ (ref. 13) and observations of $\mathrm{AB}_{2}$ Laves phases in other van der Waals compounds ${ }^{21}$, we do not believe that the present $\mathrm{N}_{2}\left(\mathrm{H}_{2}\right)_{2}$ compound represents a Laves phase. Indeed, there is only one cubic Laves phase with the $\mathrm{MgCu}_{2}$-type structure $(\mathrm{Fd} 3 \mathrm{~m})$ and the ratio of effective molecular radii does not match the criteria for optimum packing expected for such a phase. Instead, it appears more likely that $\mathrm{H}_{2}$ molecules either substitute in the $\mathrm{N}_{2}$ lattice or assume interstitial positions, leading to the distension of the unit cell in a $\delta$ - $\mathrm{N}_{2}$-like structure.

In contrast, the structure of the hexagonal solid, $\left(\mathrm{N}_{2}\right)_{6}\left(\mathrm{H}_{2}\right)_{7}$, differs entirely from the corresponding equilibrium structures in either $\mathrm{N}_{2}$ or $\mathrm{H}_{2}$ (Fig. 2). It is also unlike a previously reported $\mathrm{N}_{2}-$ $\mathrm{D}_{2}$ structure ${ }^{9}$, which was not apparent in our experiments. Instead, we identify a novel host-guest compound with $36 \mathrm{~N}_{2}$ and $42 \mathrm{H}_{2}$ molecules in a rhombohedral unit cell of $R-3 m$ space group. The lattice parameters for the conventional triple hexagonal cell are $a=b=14.273(3) \AA$, and $c=8.075(9) \AA$ at $8 \mathrm{GPa}$. Full crystallographic details are given in Supplementary Table 1. As illustrated in Fig. 2, a cage-like lattice of $\mathrm{N}_{2}$ molecules confines clusters of rotationally disordered $\mathrm{H}_{2}$ molecules. Helical chains of $\mathrm{N}_{2}$ molecules (emphasized in red, Fig. 2b) extend along the $\langle 001\rangle$ direction and confine intermediate $\mathrm{N}_{2}$ molecules in a puckered hexagonal configuration (emphasized in green). These effectively form the ends of large, open polyhedral volumes (Fig. 2c), each of which hosts $15 \mathrm{H}_{2}$ molecules (two of which are shared with the neighbouring volumes, above and below, forming channels along the $c$-axis). This host-guest configuration is radically different from most van der Waals compounds, which often assume structures according to ideal hard-sphere packing configurations (for example, $\left.\mathrm{Ar}\left(\mathrm{H}_{2}\right)_{2}, \mathrm{CH}_{4}\left(\mathrm{H}_{2}\right)_{2}, \mathrm{Xe}\left(\mathrm{O}_{2}\right)_{2}\right)^{13,16,2 \mathrm{Y}}$ or share a close structural resemblance with one of the constituents (for example, $\left(\mathrm{N}_{2}\right)_{11} \mathrm{He}$ ) (ref. 22). It is also notable that although $\mathrm{N}_{2}$ molecules maintain rotational degrees of freedom in the pure phase $\left(\delta-\mathrm{N}_{2}\right)$, here they are oriented. The presence of the guest $\mathrm{H}_{2}$ molecules and the resulting quadrupolar interactions in the novel structure favour the formation of an ordered lattice, despite the average intermolecular distances being slightly greater than in pure $\mathrm{N}_{2}$. Cage-like structures, such as this, may represent an efficient means of 'chemical compression' by enhancing confinement of the guest molecules. Because of the potential for unique interactions in this novel host-guest structure, we set out to explore the physical and chemical behaviour of $\left(\mathrm{N}_{2}\right)_{6}\left(\mathrm{H}_{2}\right)_{7}$.

Raman and IR spectroscopy reveal pressure-induced chemistry. Experiments were carried out on compression and decompression to $60 \mathrm{GPa}$ using Raman and IR spectroscopy to investigate changes in bonding in the $\left(\mathrm{N}_{2}\right)_{6}\left(\mathrm{H}_{2}\right)_{7}$ compound. Separate experiments were carried out to $160 \mathrm{GPa}$ to track changes in IR and visible absorption. The pressure evolution of the vibron modes is shown in Fig. $3 . \mathrm{H}_{2}$ exhibits three IR-active vibron modes and three Raman-active modes. The highest frequency branch agrees closely in frequency and is likely shared (both Raman- and IR-active), whereas the lower frequency branches may be ascribed to different modes. All observed modes are blueshifted with respect to pure $\mathrm{H}_{2}$ due to partial suppression of the vibrational coupling, as observed in other $\mathrm{H}_{2} /$ rare gas compounds ${ }^{23-25}$. In addition, the $\mathrm{H}_{2}$ rotational mode near $600 \mathrm{~cm}^{-1}$ is seen to persist to $50 \mathrm{GPa}$, confirming that hydrogen remains rotationally free, in contrast to its $\mathrm{N}_{2}$ host lattice (Supplementary Fig. 5). Two Raman active modes and two IR active modes are observed for $\mathrm{N}_{2}$, with vibrational frequencies that closely follow the $v_{2}$ mode of pure nitrogen and its branching with pressure. It is notable that IR activity begins for both species on crystallization. This is in stark contrast to the pure phases, where the IR modes a

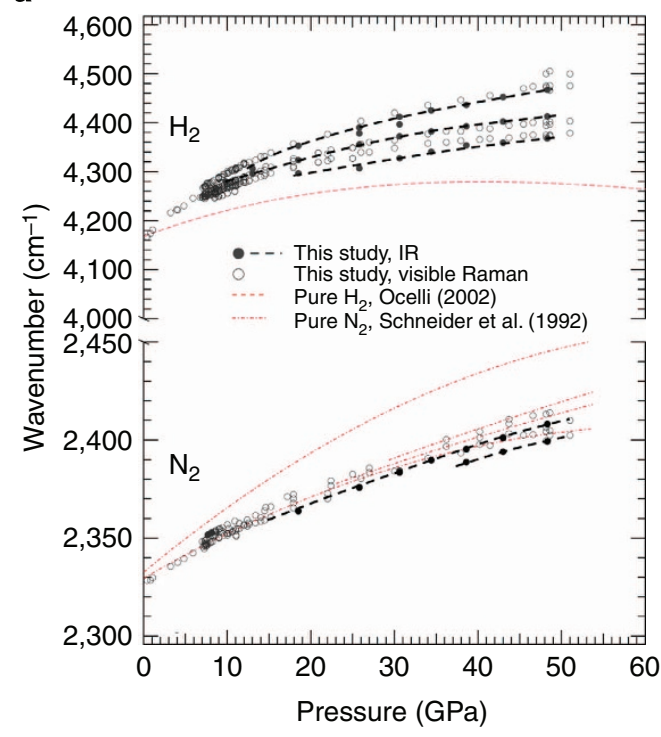

b

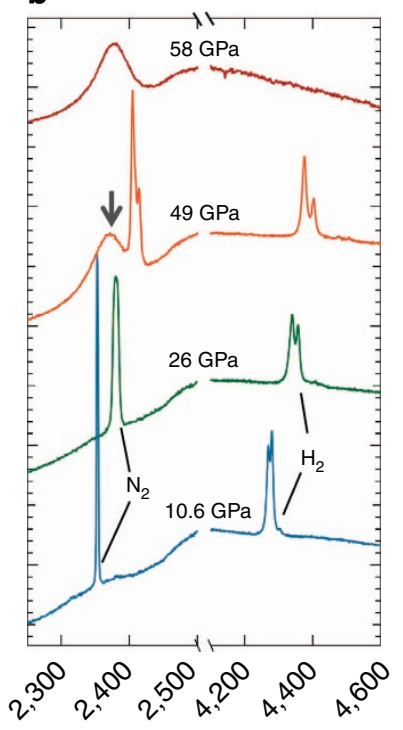

C

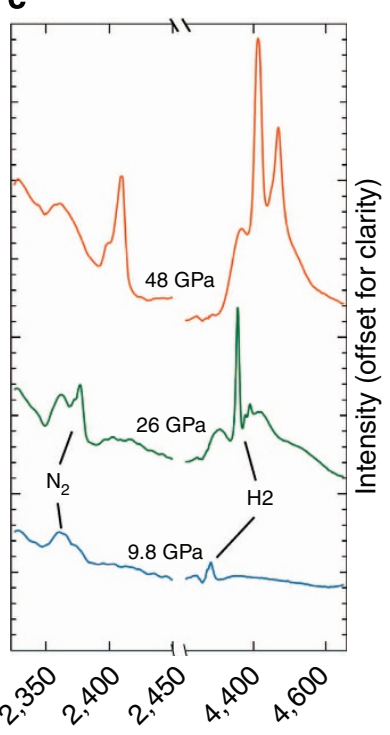

Wavenumber $\left(\mathrm{cm}^{-1}\right)$

Figure 3 | Evidence for a chemical reaction in $\left(\mathbf{N}_{\mathbf{2}}\right)_{6}\left(\mathbf{H}_{\mathbf{2}}\right)_{\mathbf{7}}$ near $\mathbf{5 0} \mathbf{~ G P a}$. (a) Evolution of Raman and infrared vibrons for the $\left(\mathrm{N}_{2}\right)_{6}\left(\mathrm{H}_{2}\right)_{7}$ inclusion compound $\left(54 \mathrm{~mol} \% \mathrm{H}_{2}\right.$ ). Red dashed curves are from refs 25,45 . (b) Visual Raman data taken on compression show the appearance of a new, broad feature at $2,370 \mathrm{~cm}^{-1}$, which appears near $47 \mathrm{GPa}$ and grows at the expense of the $\mathrm{v}_{2}$ mode of $\mathrm{N}_{2}$ with increasing pressure (indicated by an arrow). Following the appearance of this mode, the $\mathrm{N}_{2}$ and $\mathrm{H}_{2}$ vibrons diminish and then disappear with time (as shown at $58 \mathrm{GPa}$ ), suggesting a (sluggish) transition to an amorphous phase in which the previously inert molecules have reacted. (c) Infrared absorption data, also on compression, were collected on a separate sample. IR vibrons also disappear with time and with increasing pressure as a new, high-pressure phase forms from the previously crystalline solid. 
appear at 20 and $215 \mathrm{GPa}$ for $\mathrm{N}_{2}$ and $\mathrm{H}_{2}$, respectively. This indicates an induced dipolar moment with increasing pressure, not typically present in a homonuclear diatomic molecule (Fig. 3c).

At $49 \mathrm{GPa}$, a marked change in vibron signature is observed. The $\mathrm{H}_{2}$ symmetric stretching modes disappear, whereas the $\mathrm{N}_{2}$ Raman modes give way to a broad, lower-frequency feature (Fig. 3b), suggesting a possible amorphization. The amorphous state was later confirmed by the absence of X-ray diffraction peaks above the transition pressure. Despite this significant change, the solid appears to remain macroscopically intact with no visible phase separation. The transition occurs sluggishly throughout the sample, going to completion in a matter of hours and can be overdriven by as much as $15 \mathrm{GPa}$ under very rapid compression. In time, however, a very broad feature appears in the Raman data in regions associated with $\mathrm{N}-\mathrm{H}$ symmetric and antisymmetric stretch modes $(\sim 2,900-3,600)$, suggesting disruption of the strong $\mathrm{N}_{2}$ triple bond and the onset of a chemical transformation in the amorphous, reacted solid (Fig. 4).

To better understand this transition, we probed the forward and reverse transformations in several loadings, collecting IR, Raman and visible-absorption measurements. Typical IR and Raman spectra obtained on decompression of the amorphous solid are shown in Fig. 4a,b, and suggest that the high-pressure phase has an ionic character. Most significantly, we note that IR absorption is enhanced with a broad absorption feature between $\sim 2,100-2,900 \mathrm{~cm}^{-1}$ (shaded region, Fig. 4a). These spectra bear a strong resemblance to recent experiments and theory on the ammonium amide phase $\left(\mathrm{NH}_{4}^{+} / \mathrm{NH}_{2}^{-}\right)$, shown to result from the auto-ionization of $\mathrm{NH}_{3}$, albeit at much more extreme conditions ${ }^{26-28}$. The broadband centered near $1,500 \mathrm{~cm}^{-1}$ is consistent with the bending modes of $\mathrm{NH}_{4}^{+}$and $\mathrm{NH}_{2}^{-}$. With increasing pressure, a strong visible absorption band is also observed between $\sim 400-500 \mathrm{~nm}$, resulting in a darkening of the sample (Supplementary Fig. 6). Together with the IR data, this suggests the onset of increased charge transfer between nitrogen and hydrogen, signalling the formation of ionized $\mathrm{NH}_{3}$ in an amorphous $\mathrm{N}_{2}$ structure.

On decompression, the visible absorption band blueshifts and decreases in intensity, accompanied by distinct structuring of $\mathrm{N}$ $\mathrm{H}$ Raman and IR vibrational modes. The ionic character of the sample appears diminished below $\sim 40 \mathrm{GPa}$. The breadth and relative intensity of the Raman modes are potentially consistent with an $\mathrm{NH}_{3}$-rich solid at higher pressures (Fig. 4b), however with decreasing pressure the stretching modes shift to lower frequency and become increasingly structured, evolving towards the spectral signature of $\mathrm{N}_{2} \mathrm{H}_{4}$ hydrazine $)^{29,30}$. Below $9 \mathrm{GPa}$, the $\mathrm{N}_{2}$ vibron band progressively transforms into two well-resolved peaks close to those of pure $\mathrm{N}_{2}$. The solid only slightly deforms before a clear phase separation between liquid $\mathrm{N}_{2}$ and solid $\mathrm{N}_{2} \mathrm{H}_{4}$, well below the melting pressure of the original $\left(\mathrm{N}_{2}\right)_{6}\left(\mathrm{H}_{2}\right)_{7}$ compound (Supplementary Fig. 7) confirming that a reaction has taken place. Excess $\mathrm{H}_{2}$ may be present but was not detected.

\section{Discussion}

The structure of the $\left(\mathrm{N}_{2}\right)_{6}\left(\mathrm{H}_{2}\right)_{7}$ compound appears to facilitate intermolecular interactions sufficient to break down the strong $\mathrm{N} \equiv \mathrm{N}$ triple bond and promote $\mathrm{N}-\mathrm{H}$ bonding above $50 \mathrm{GPa}$, resulting in chemical behaviour reminiscent of that seen in other nitrogen compounds at much more extreme conditions. Indeed, the auto-ionization of $\mathrm{NH}_{3}$ to form ammonium amide $\left(\mathrm{NH}_{4}^{+}\right.$/ $\mathrm{NH}_{2}^{-}$) was seen at pressures more than twice as high as our observations ${ }^{27,28}$. Mixtures of nitrogen and oxygen have also been shown to transform from molecular compounds to ionic solids (nitrosonium nitrate), though only at high temperatures ${ }^{31}$ or
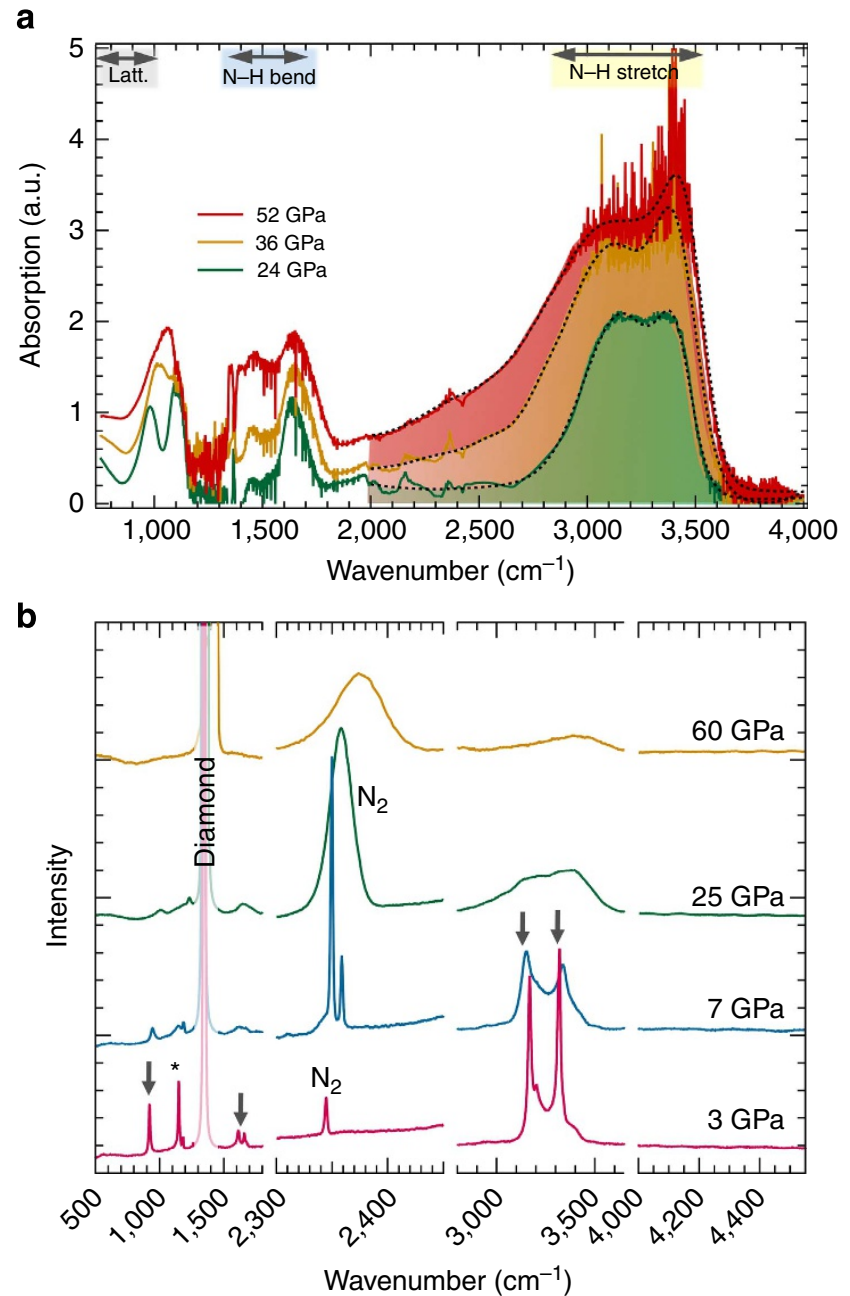

Figure 4 | Transition from van der Waals to ionic interactions in $\left(\mathbf{N}_{\mathbf{2}} \mathbf{~}_{\mathbf{6}}\left(\mathbf{H}_{\mathbf{2}} \mathbf{~}_{\mathbf{7}}\right.\right.$. (a) Following the pressure-induced chemical reaction in $\left(\mathrm{N}_{2}\right)_{6}\left(\mathrm{H}_{2}\right)_{7}$ near $50 \mathrm{GPa}$, a broad band is observed in infrared absorption spectra above $2,000 \mathrm{~cm}^{-1}$ (shaded), suggesting the presence of $\mathrm{NH}_{4}^{+} / \mathrm{NH}_{2}^{-}$in the high-pressure phase and the breakdown of van der Waals interactions in the solid. With decreasing pressure, $\mathrm{N}-\mathrm{H}$ stretching modes become increasingly resolved and resemble those of $\mathrm{NH} 3$ (green $)^{28}$, suggesting reaction of the species. (b) Visible Raman data demonstrate extreme broadening and slight redshift of the principle $\mathrm{N}_{2}$ stretch mode $\left(2,300-2,400 \mathrm{~cm}^{-1}\right)$ in the high-pressure phase, suggesting an amorphous state. Broad $\mathrm{N}-\mathrm{H}$ stretch modes (already evident at high pressure, $3,000-3,500 \mathrm{~cm}^{-1}$ ) become increasingly structured on decompression and are consistent with the recovery of a mixture of pure $\mathrm{N}_{2}$ and hydrazine $\left(\mathrm{N}_{2} \mathrm{H}_{4}\right)^{29,46}$. The peak at $\sim 1,100 \mathrm{~cm}^{-1}$ is consistent with an $\mathrm{N}-\mathrm{N}$ stretch mode (indicated by a ${ }^{\star}$ ), whereas the others (indicated by grey arrows) may be assigned to $\mathrm{N}-\mathrm{H}$ bending, stretching and deformational modes.

when subjected to laser or X-ray irradiation ${ }^{32}$. Similarly, pure $\mathrm{N}_{2}$ exhibits an amorphous, narrow band-gap, nonmolecular phase $(\eta)$ but only at pressures above $150 \mathrm{GPa}^{1,5,33-35}$. The present results demonstrate the possibility of manipulating the topochemistry of simple mixtures to markedly reduce the thresholds for pressure-induced reactions, leading to behaviour otherwise observed at much higher pressures and/or temperatures. Such high-pressure phases have tremendous potential for inducing transitions from predominantly intra- to intermolecular interactions, well within the stability regimes of the end-member constituents, as demonstrated by the observed shift from van der Waals to ionic interactions in $\left(\mathrm{N}_{2}\right)_{6}\left(\mathrm{H}_{2}\right)_{7}$. This 
is a promising avenue for exploring 'tunable' pressure-induced chemistry and potentially lowering activation barriers for favourable reactions in other molecular systems.

We have presented a preliminary nitrogen-hydrogen binary phase diagram, which shows evidence for two new van der Waals compounds. $\left(\mathrm{N}_{2}\right)_{6}\left(\mathrm{H}_{2}\right)_{7}$ was characterized and shown to undergo a change from van der Waals to ionic interactions coincident with a transformation to an amorphous phase near $50 \mathrm{GPa}$. On decompression, spectroscopic data show that $\mathrm{N}-\mathrm{H}$ bonds evolve in the reacted solid, forming ammonium amide at high pressures and then leading to the formation of $\mathrm{N}_{2} \mathrm{H}_{4}$ in equilibrium with liquid $\mathrm{N}_{2}$ on melting. These results demonstrate that very interesting molecular arrangements such as the remarkable hostguest structure of $\left(\mathrm{N}_{2}\right)_{6}\left(\mathrm{H}_{2}\right)_{7}$ can be obtained through the pressure-driven formation of van der Waals compounds. Additional stimuli (for example, photochemistry by irradiation) may further facilitate such reactions and if similar structures can be stabilized and recovered, they may represent a means of engineering solids with desirable technological applications. Other such structures may also present unique opportunities for the chemical confinement of molecular $\mathrm{H}_{2}$. The stability and properties of $\left(\mathrm{N}_{2}\right)_{6}\left(\mathrm{H}_{2}\right)_{7}$ at low temperature will be the subject of future studies, and we hope that this work will motivate experimental and theoretical structural searches in other binary mixtures.

\section{Methods}

Sample preparation. Nitrogen-hydrogen mixtures were prepared in membrane DACs at either $20 \mathrm{MPa}$ from standard bottles or $140 \mathrm{MPa}$ using a gas-loading compressor. Partial gas pressures for a given concentration were calculated using a second-order virial correction to account for interaction of mixing and mixtures were left to equilibrate for at least $24 \mathrm{~h}$ before loading. Uncertainties in concentration are $<1 \mathrm{~mol} \%$. Low-pressure experiments $(<30 \mathrm{GPa})$ used CuBe gaskets and Boehler-Almax-type bevelled anvils with $300 \mu \mathrm{m}$ culets. High pressures $(>30 \mathrm{GPa}$ ) were achieved using Au-lined rhenium gaskets and diamond culets ranging from 40 to $100 \mu \mathrm{m}$ with the sample diameter being $\sim 1 / 3-1 / 2$ that of the culet in all cases. Single crystal samples were grown under quasi-hydrostatic conditions from solid-fluid equilibrium by pressurization at room temperature and pressures were determined using the ruby fluorescence scale and/or the frequency shift of the first-order diamond phonon ${ }^{36-38}$.

\section{Spectroscopy measurements. Visible Raman spectroscopy was performed in} backscattering geometry and the $487.986 \mathrm{~nm}$ line from a continuous Ar laser, focused to $\sim 3 \mu \mathrm{m}$ at the sample. Data were recorded using a gain-amplified charge-coupled device (CCD) coupled to an Andor spectrometer, resulting in a spectral resolution of $\sim 2 \mathrm{~cm}^{-1}$. Synchrotron IR spectroscopy was carried out on the SMIS beamline at the Soleil synchrotron between 650 and $8,000 \mathrm{~cm}^{-1}$ using a coupled Fourier Transform Infrared (FTIR) spectrometer ${ }^{39}$. Additional IR measurements were conducted using a custom benchtop microspectroscopy system $^{40}$. The empty DAC was used as a reference for treatment of the absorption data.

\section{X-ray diffraction and structural analysis. Structural information was obtained} from angle-dispersive single-crystal X-ray diffraction carried out on beamline ID09 (ref. 41) of the European Synchrotron Radiation Facility (Grenoble, France), using a focused $(\sim 20 \mu \mathrm{m})$ monochromatic beam at $\lambda=0.4147 \AA(29.8 \mathrm{keV})$. Data were recorded on a MAR 555 flat-panel detector calibrated with a Si standard. The sample was rotated in $0.5^{\circ}$ oscillations over a range of $58^{\circ}$ about the vertical axis during collection. CrysalisPro (Oxford Diffraction) was used to analyze the images and index reflections. The structure of the $\left(\mathrm{N}_{2}\right)_{6}\left(\mathrm{H}_{2}\right)_{7}$ compound was solved using the $a b$ initio charge-flipping algorithm, Superflip ${ }^{42}$. SHELXL ${ }^{43}$ and Olex2 (ref. 44) software packages were used for structural refinement. Complete details of the structural analysis are provided in the Supplementary Discussion and Supplementary Data 1 . These techniques, together with visual microscopic observation, provide a complete suite of data on the vibrational, structural and macroscopic characteristics of the sample.

\section{References}

1. Gregoryanz, E. et al. High P-T transformations of nitrogen to 170GPa. J. Chem. Phys. 126, 184505 (2007).

2. McMahon, J., Morales, M., Pierleoni, C. \& Ceperley, D. M. The properties of hydrogen and helium under extreme conditions. Rev. Mod. Phys. 84, 1607-1653 (2012).
3. Eremets, M., Gavriliuk, A. G., Trojan, I. A., Dzivenko, D. A. \& Boehler, R. Single-bonded cubic form of nitrogen. Nat. Mater. 3, 558-563 (2004).

4. Ciezak, J. in Metastable Polymeric Nitrogen: the Ultimate Green High-Energy Density Material. The U.S. Army Research Laboratory Report ARL-TR-4478 (2008).

5. Lipp, M. J. et al. Transformation of molecular nitrogen to nonmolecular phases at megabar pressures by direct laser heating. Phys. Rev. B 76, 014113 (2007).

6. Zahariev, F., Dudiy, S. V., Hooper, J., Zhang, F. \& Woo, T. K. Systematic method to new phases of polymeric nitrogen under high pressure. Phys. Rev. Lett. 97, 155503 (2006).

7. Loubeyre, P., Occelli, F. \& Le Toullec, R. Optical studies of solid hydrogen to 320GPa and evidence for black hydrogen. Nature 416, 613-617 (2002).

8. Zha, C., Liu, Z., Ahart, M., Boehler, R. \& Hemley, R. J. High-pressure measurements of hydrogen phase IV using synchrotron infrared spectroscopy. Phys Rev. Lett. 110, 217402 (2013).

9. Kim, M. \& Yoo, C.-S. Highly repulsive interaction in novel inclusion $\mathrm{D}_{2}-\mathrm{N}_{2}$ compound at high pressure: Raman and X-ray evidence. J. Chem. Phys. 134, 044519 (2011).

10. Ciezak, J., Jenkins, T. A. \& Hemley, R. J. in Proceedings Shock Compression of Condensed Matter. eds. Elert, M. L., Butler, W. T., Anderson, W. W. \& Proud, W. G.) 1291-1294 (American Institute of Physics, 2009).

11. Jena, P. Materials for hydrogen storage: past, present, and future. J. Phys. Chem. Lett 2, 206-211 (2011).

12. Ashcroft, N. W. Hydrogen dominant metallic alloys: high temperature superconductors? Phys Rev. Lett. 92, 187002 (2004).

13. Loubeyre, P., LeToullec, R. \& Pinceaux, J. P. Compression of $\operatorname{Ar}\left(\mathrm{H}_{2}\right)_{2}$ up to 175GPa: a new path for the dissociation of molecular hydrogen? Phys. Rev. Lett. 72, 1360-1363 (1994).

14. Vos, W. L. \& Schouten, J. A. The stability of van der Waals compounds at high pressures. Fiz. Nizk. Temp. (Low Temp. Phys.) 19, 481-485 (1993).

15. Loubeyre, P. \& Le Toullec, R. Stability of $\mathrm{O}_{2} / \mathrm{H}_{2}$ mixtures at high pressure. Nature 378, 44-46 (1995).

16. Somayazulu, M., Finger, L. W., Hemley, R. J. \& Mao, H. K. High-pressure compounds in methane-hydrogen mixtures. Science 271, 1400-1402 (1996).

17. Somayazulu, M. et al. Pressure-induced bonding and compound formation in xenon-hydrogen solids. Nat. Chem. 2, 50-53 (2009).

18. Strobel, T., Somayazulu, M. \& Hemley, R. J. Novel pressure-induced interactions in silane-hydrogen. Phys. Rev. Lett. 103, 065701 (2009).

19. Cromer, D. T., Mills, R. L., Schiferl, D. \& Schwalbe, L. A. The structure of $\mathrm{N}_{2}$ at 49kbar and 299K. Acta Crystallogr. B37, 8-11 (1981).

20. Stinton, G. W., Loa, I., Lundegaard, L. F. \& McMahon, M. The crystal structures of delta and delta* nitrogen. J. Chem. Phys. 131, 104511 (2009).

21. Weck, G., Dewaele, A. \& Loubeyre, P. Oxygen/noble gas binary phase diagrams at 296K and high pressures. Phys. Rev. B 82, 014112 (2010).

22. Ninet, S., Weck, G., Loubeyre, P. \& Datchi, F. Structural and vibrational properties of the van der Waals compound $\left(\mathrm{N}_{2}\right)_{11} \mathrm{He}$ up to $135 \mathrm{GPa}$. Phys. Rev. B. 83, 134107 (2011).

23. Loubeyre, P., Le Toullec, R. \& Pinceaux, J. P. Properties of $\mathrm{H}_{2}$ under strong compression in a Ne matrix. Phys. Rev. Lett. 67, 3271-3274 (1991).

24. Loubeyre, P., Toullec, R. \& Pinceaux, J. P. Raman measurements of the vibrational properties of $\mathrm{H}_{2}$ as a guest molecule in dense helium, neon, argon, and deuterium systems up to 40GPa. Phys. Rev. B 45, 12844-12853 (1992).

25. Occelli, F. Physique de l'Hydrogene a Haute Pression, PhD thesis, Univ. Paris VI (2002).

26. Pickard, C. J. \& Needs, R. J. Highly compressed ammonia forms an ionic crystal. Nat. Mater. 7, 775-779 (2008).

27. Palasyuk, T. et al. Ammonia as a case study for the spontaneous ionization of a simple hydrogen-bonded compound. Nat. Commun 5, 3460 (2014).

28. Ninet, S. et al. Experimental and theoretical evidence for an ionic crystal of ammonia at high pressure. Phys. Rev. B 89, 174103 (2014).

29. Jiang, S. et al. Hydrogen Bond in Compressed Solid Hydrazine. J. Phys Chem. C 118, 3236-3243 (2014).

30. Ninet, S., Datchi, F., Saitta, A. M., Lazzeri, M. \& Canny, B. Raman Spectrum of Ammonia IV. Phys. Rev. B 74, 104101 (2006).

31. Somayazulu, M. et al. Novel broken symmetry phase from $\mathrm{N}_{2} \mathrm{O}$ at high pressures and high temperatures. Phys. Rev. Lett. 87, 135504 (2001).

32. Sihachakr, D. \& Loubeyre, P. High-pressure transformation of $\mathrm{N}_{2} / \mathrm{O}_{2}$ mixtures into ionic compounds. Phys. Rev. B 74, 064113 (2006).

33. Gregoryanz, E., Goncharov, A. F., Hemley, R. J. \& Mao, H. K. High-pressure amorphous nitrogen. Phys. Rev. B 64, 502103 (2001).

34. Gregoryanz, E. et al. Raman, infrared, and X-ray evidence for new phases of nitrogen at high pressures and temperatures. Phys. Rev. B 66, 224108 (2002).

35. Goncharov, A. F., Gregoryanz, E., Mao, H. K., Liu, Z. \& Hemley, R. J. Optical evidence for a nonmolecular phase of nitrogen above 150GPa. Phys. Rev. Lett. 85, 1262-1265 (2000).

36. Piermarini, G. J., Block, S., Barnett, J. D. \& Forman, R. A. Calibration of the pressure dependence of the $\mathrm{R}_{1}$ ruby fluorescence line to 195kbar. J. Appl. Phys 46, 2774-2780 (1975). 
37. Datchi, F. et al. Optical pressure sensors for high-pressure-high-temperature studies in a diamond anvil cell. High Press. Res 27, 447-483 (2007).

38. Akahama, Y. \& Kawamura, H. High-pressure Raman spectroscopy of diamond anvils to 250GPa: Method for pressure determination in the multimegabar pressure range. J. Appl. Phys. 96, 3748-3751 (2004).

39. Dumas, P. et al. Synchrotron Infrared Microscopy at the French Synchrotron facility SOLEIL. Infrared Phys. Techn. 49, 152-160 (2006).

40. Andre, R. in Micro Spectroscopie Raman Par Transformée de Fourier Proche Infrarouge (Editions Universitaires Europeennes, 2011).

41. Merlini, M. \& Hanfland, H. Single-crystal diffraction at megabar conditions by synchrotron radiation. High Press. Res. 33, 511-522 (2013).

42. Palatinus, L. \& Chapuis, G. SUPERFLIP - a computer program for the solution of crystal structures by charge flipping in arbitrary dimensions. J. Appl. Cryst. 40, 786-790 (2007).

43. Sheldrick, G. A short history of SHELX. Acta Cryst. A 64, 112-122 (2008).

44. Dolomanov, O. V., Bourhis, L. J., Gildea, R. J., Howard, J. A. K. \& Puschmann, H. OLEX2: a complete structure solution, refinement and analysis program. J. Appl. Cryst. 42, 339-341 (2009).

45. Schneider, H., Hafner, W., Wokaun, A. \& Olijnyk, H. Room temperature Raman scattering studies of external and internal modes of solid nitrogen at pressures $8<\mathrm{P}<54 \mathrm{GPa}$. J. Chem. Phys. 96, 8046-8053 (1992).

46. Chellappa, R., Dattelbaum, D., Daemen, L. \& Liu, Z. High pressure spectroscopic studies of hydrazine (N2H2), Proceedings 18th APS-SCCM and 24th AIRAPT, J. Phys. Conf. Series 500, 052008, IOP Publishing (2014).

\section{Acknowledgements}

We thank Mr Ramesh André and Dr Florent Ocelli for their assistance with sample preparation, experiments and helpful discussions.

\section{Author contributions}

D.K.S., G.W. and P.L. designed the project. D.K.S. and G.W. conducted the experiments and analyzed the data. F.D. assisted with structural analyses and interpretation. P.D. and M.H. assisted with synchrotron experiments. D.K.S., G.W., P.L. and F.D. wrote the manuscript. All authors discussed results.

\section{Additional information}

Supplementary Information accompanies this paper at http://www.nature.com/ naturecommunications

Competing financial interests: The authors declare no competing financial interests.

Reprints and permission information is available online at http://npg.nature.com/ reprintsandpermissions/

How to cite this article: Spaulding, D. K. et al. Pressure-induced chemistry in a nitrogenhydrogen host-guest structure. Nat. Commun. 5:5739 doi: 10.1038/ncomms6739 (2014). 